\title{
Schema Matching over Relations, Attributes, and Data Values
}

\author{
Aibo Tian, Mayank Kejriwal, Daniel P. Miranker \\ Department of Computer Science \\ The University of Texas at Austin, USA \\ \{atian, mayankkejriwal\}@utexas.edu, miranker@cs.utexas.edu
}

\begin{abstract}
Automatic schema matching algorithms are typically only concerned with finding attribute correspondences. However, real world data integration problems often require matchings whose arguments span all three types of elements in relational databases: relation, attribute and data value. This paper introduces the definitions and semantics of three additional correspondence types. These correspondences cover the higher-order mappings identified in a seminal paper by Krishnamurthy, Litwin, and Kent. It is shown that these correspondences can be automatically translated to tuple generating dependencies (tgds), and thus this research is compatible with data integration applications that leverage tgds.

Two methods for automatically identifying these correspondences are developed. One requiring a limited number of duplicates across data sources. The other is a general instance-based method with no such requirement. Experiments conducted on four real world data sets demonstrate the effectiveness of the two methods.
\end{abstract}

\section{Categories and Subject Descriptors}

H.2.5 [Database Management]: Heterogeneous Databases

\section{Keywords}

Schema Matching, Compound Correspondence, Higher-order Schema Matching

\section{INTRODUCTION}

Schema matching is an essential component of information integration systems [3, 4]. Most existing automatic schema matchers define the matching problem as finding attribute correspondences. This definition has limitations in practice. In a seminal paper, Krishnamurthy, Litwin, and Kent provide concrete examples to show that due to schematic discrepancies, interoperability of databases requires higher order capabilities over both metadata and data [18]. They

Permission to make digital or hard copies of all or part of this work for personal or classroom use is granted without fee provided that copies are not made or distributed for profit or commercial advantage and that copies bear this notice and the full citation on the first page. To copy otherwise, to republish, to post on servers or to redistribute to lists, requires prior specific permission and/or a fee.

WOODSTOCK' 97 El Paso, Texas USA

Copyright 20XX ACM X-XXXXX-XX-X/XX/XX ...\$15.00.

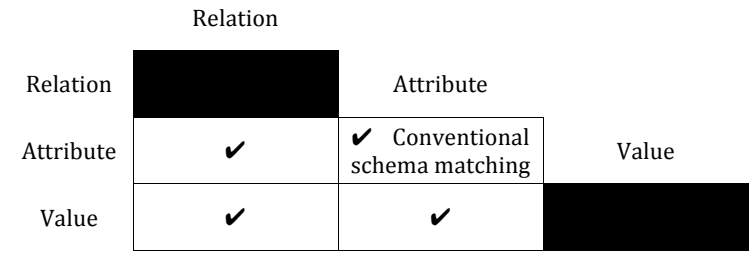

Figure 1: Six possible correspondence types. The checked correspondence types are detailed in this paper. The definition of correspondence is based on a given pair of relations, so correspondence between relations is ignored. Correspondence between values does not involve metadata; hence it is not considered.

also state that schematic discrepancies are frequent. Figure 2 shows the three stock price databases from their paper, which are asserted in commercial use [18]. The three databases contain similar information, but are organized differently. For example, the stock "IBM" is a [D]ata value in database $\mathrm{D}$, an attribute $([\mathrm{C}]$ olumn $)$ in database $\mathrm{C}$, and a $[R]$ elation in database $R$. Throughout the paper, an element in a database is denoted as its name prepended by the database name and a colon, e.g. "C:IBM".

A relational database contains three types of elements: relation, attribute, and data value. Any pair of elements can form a correspondence, resulting in six possible correspondence types (see Figure 1). The conventional schema matching definition only considers one possibility. We call the correspondences between elements of the same type as same-type correspondences, and between elements of different types as different-type correspondences.

Different-type correspondences are frequently required in real world data integration applications. Take ecommerce domain with car listings for example (see Figure 4 for our real data set in commercial use). Properties of cars, such as mileage and color, can be designed as attributes in carspecific databases. They can be also designed as data values, if the databases are general-purpose that does not have prior information of products. Integrating these two kinds of databases is similar to integrating database $\mathrm{D}$ and $\mathrm{C}$ in Figure 2, which requires matchings between attributes and data values.

The same-type correspondence between two attributes $a$ and $b$ has clear semantics. If two tuples $s$ and $t$ satisfy the correspondence, the value of $a$ in $s$ equals the value of $b$ in $t$. The semantics of different-type correspondences are not clear. In Figure 2, correspondence between attribute 


Stock
\begin{tabular}{|l|l|l|}
\hline date & stkCode & clsPrice \\
\hline $1 / 2 / 13$ & AAPL & 549 \\
$1 / 2 / 13$ & IBM & 196 \\
\hline
\end{tabular}

(a) Database D: stock as [D]ata value
Stock

\begin{tabular}{|l|l|l|l}
\hline date & AAPL & IBM & GOOG \\
\hline $1 / 2 / 13$ & 549 & 196 & 723 \\
$1 / 3 / 13$ & 542 & 195 & 723 \\
& & &
\end{tabular}

(b) Database C: stock as attribute ([C]olumn)

\section{IBM}

\begin{tabular}{|l|l|}
\hline date & clsPrice \\
\hline $1 / 2 / 13$ & 196 \\
$1 / 3 / 13$ & 195 \\
\hline
\end{tabular}

(c) Database R: stock as [R]elation

Figure 2: Example databases of stock prices.

"C:IBM" and data value "D:IBM" indicates a relationship between static metadata and dynamic data. The meaning of such a relationship is not obvious.

We observe that a different-type correspondence takes on clear semantics if combined with an appropriate same-type correspondence. For example, consider the different-type correspondence between attribute "C:IBM" and data value "D:IBM". When combined with the same-type correspondence between the data values of attributes "C:IBM" and "D:clsPrice", it represents the matching of IBM stock prices. "IBM" and its stock price "196" in both databases can now be matched by specifying this compound correspondence.

In this work, the definitions and semantics of three types of compound correspondences are introduced. The compound correspondence types are named by their differenttype correspondences as attribute-value, relation-attribute, or relation-value. The checked cells (except correspondence between attributes) in Figure 1 represent these differenttype correspondences.

Two algorithms to automatically generate compound correspondences are provided. Both algorithms exploit database instances. One algorithm generates correspondences using duplicates in the two databases, an idea demonstrated to be effective in the DUMAS system [5]. The other algorithm is more general in that it does not use duplicates. Experiments conducted on four real world data sets demonstrate the effectiveness of the two methods comparing with DUMAS and an instance-based schema matcher.

A common schema mapping representation in information integration applications is tuple-generating dependency (tgd). To demonstrate that compound correspondences can be used in both data exchange and data integration applications, we propose an algorithm to automatically translate these correspondences to tgds with constants. We slightly change the chase algorithm [20] to generate universal solutions based on these tgds for data exchange. Further more, discussion of applying existing query rewriting algorithms to these tgds for data integration is provided.

\section{DEFINITIONS}

This paper considers relational data models. A schema is a finite set of relations. We denote schemas as $\mathcal{A}, \mathcal{B}$, and relations as $A, B$. A relation is a finite set of attributes. A tuple is a finite set that contains a value for each attribute. Attributes are represented as $a, b$, and tuples are represented as $s, t$. Given an attribute $a$ and a tuple $t$, the value of $a$ in $t$ is denoted as $t^{a}$.

In order to define compound correspondences, we introduce the notion of database element set. Intuitively, the database element set of a relation $A$ is the set of all elements of $A$ that can occur in correspondences. A database element set contains relation names, attribute names, and data val- ues. Introducing data values in correspondences raises the issue of how to represent data values. A straightforward way is to use actual data values. However, data values are dynamic and can change frequently. A second way, which is used in this paper, is to use a symbolic representation. We use notation $\bar{a}$ to represent the set of all possible data values of attribute $a$. For example, $\overline{\mathrm{D}: \text { stkCode }}$ represents "AAPL", "IBM" and other stock codes. The program using the correspondences needs to match the exact data values.

Definition 2.1 (DATABASE ElEment SET). Given a relation $A$, and the set of attributes $\left\{a_{i}\left|a_{i} \in A, i=1, \ldots,\right| A \mid\right\}$, the database element set is $\Theta_{A}$, where $\Theta_{A}=\{A\} \cup\left\{a_{i} \mid a_{i} \in\right.$ $A\} \cup\left\{\overline{a_{i}} \mid a_{i} \in A\right\}$. A is interchangeably used to represent the name of relation $A$ and the set of all attributes in $A$, and $\overline{a_{i}}$ is the symbol representing the set of all possible data values of attribute $a_{i}$.

To define compound correspondence, we first define primitive correspondence as a correspondence between two elements in the database element sets.

Definition 2.2 (PRIMITIVE CORRESPONDENCE). Given two relations $A$ and $B$, a primitive correspondence is a pair $\left(e_{1}, e_{2}\right)$, where $e_{1} \in \Theta_{A}$, and $e_{2} \in \Theta_{B} . \Theta_{A}$ and $\Theta_{B}$ are database element sets of $A$ and $B$, respectively.

A compound correspondence is a pair of primitive correspondences, with the constraint that the second element is always defined as a primitive correspondence between data values.

DeFinition 2.3 (COMPOUnd CORRESPOndence). Given two relations $A$ and $B$, the three types of compound correspondences are defined as follows:

- Attribute-value correspondence: for an attribute $a \in A$ and attributes $b_{1}, b_{2} \in B$, an attribute-value correspondence is a pair $\left(\left(a, \overline{b_{1}}\right),\left(\bar{a}, \overline{b_{2}}\right)\right)$, where $\left(a, \overline{b_{1}}\right)$ and $\left(\bar{a}, \overline{b_{2}}\right)$ are primitive correspondences;

- Relation-attribute correspondence: for an attribute a $\in$ $A$ and an attribute $b \in B$, a relation-attribute correspondence is a pair $((A, b),(\bar{a}, \bar{b}))$, where $(A, b)$ and $(\bar{a}, \bar{b})$ are primitive correspondences;

- Relation-value correspondence: for an attribute $a \in$ $A$ and attributes $b_{1}, b_{2} \in B$, a relation-value correspondence is a pair $\left(\left(A, \overline{b_{1}}\right),\left(\bar{a}, \overline{b_{2}}\right)\right)$, where $\left(A, \overline{b_{1}}\right)$ and $\left(\bar{a}, \overline{b_{2}}\right)$ are primitive correspondences.

Figure 3 highlights some compound correspondences in Figure 2. ((C:IBM, $\overline{\mathrm{D}: \text { stkCode }}),(\overline{\mathrm{C}: \mathrm{IBM}}, \overline{\mathrm{D}: \text { clsPrice }}))$ is an attribute-value correspondence. A relation-attribute correspondence is ((R:IBM, C:IBM $),(\overline{\mathrm{R}: c l s P r i c e}, \overline{\mathrm{C}: \mathrm{IBM}}))$, and 


\begin{tabular}{|c|c|c|c|c|c|c|}
\hline \multicolumn{4}{|l|}{ Stock } & \multicolumn{3}{|l|}{ Stock } \\
\hline date & AAPL & IBM & GOOG & date & stkCode & clsPrice \\
\hline \begin{tabular}{|l|}
$1 / 2 / 13$ \\
\end{tabular} & 549 & 196 & 723 & $1 / 2 / 13$ & AAPL & 549 \\
\hline $1 / 3 / 13$ & 542 & 195 & 723 & $1 / 2 / 13$ & IBM & 196 \\
\hline
\end{tabular}

(a) Attribute-value:((C:IBM, $\overline{\text { D:stkCode }}),(\overline{\mathrm{C}: I B M}, \overline{D: c l s P r i c e}))$

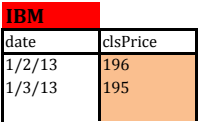

Stock
\begin{tabular}{|l|l|l|l|}
\hline date & AAPL & IBM & GOOG \\
\hline $1 / 2 / 13$ & 549 & 196 & 723 \\
$1 / 3 / 13$ & 542 & 195 & 723 \\
\hline
\end{tabular}

(b) Relation-attribute:((R:IBM, C:IBM), ( $\overline{\mathrm{R}: \mathrm{clsPrice}}, \overline{\mathrm{C}: \mathrm{IBM}}))$
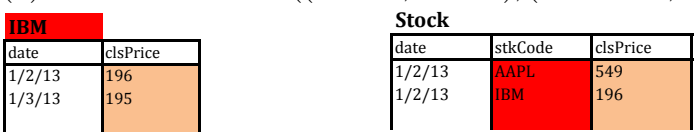

(c) Relation-value:((R:IBM, $\overline{\mathrm{D}: \text { stkCode }}),(\overline{\mathrm{R}: \text { clsPrice }}, \overline{\mathrm{D}: \text { clsPrice }}))$

Figure 3: Highlights illustrate compound correspondences.

((R:IBM, $\overline{\mathrm{D}: \text { stkCode }}),(\overline{\mathrm{R}: \text { clsPrice }}, \overline{\mathrm{D} \text { :clsPrice }}))$ is a relationvalue correspondence.

The semantics of compound correspondences are now defined. For completeness, the semantics of the conventional attribute-attribute correspondence are also presented. The notation in Definition 2.3 is employed. $s$ and $t$ represent tuples of relations $A$ and $B$. The value of an attribute $a$ in $s$ is denoted $s^{a}$.

- Attribute-attribute correspondence $(a, b)$ $s$ and $t$ satisfy correspondence $(a, b)$, if $s^{a}=t^{b}$.

Example: In Figure 2, (C:date, D:date) is an attributeattribute correspondence. The first tuple of database $\mathrm{C}$ and the first tuple of database D satisfy the correspondence.

- Attribute-value correspondence $\left(\left(a, \overline{b_{1}}\right),\left(\bar{a}, \overline{b_{2}}\right)\right)$

$s$ and $t$ satisfy correspondence $\left(\left(a, \overline{b_{1}}\right),\left(\bar{a}, \overline{b_{2}}\right)\right)$, if $a=$ $t^{b_{1}}$ and $s^{a}=t^{b_{2}}$.

Example: The first tuple of database $\mathrm{C}$ and the second tuple of database D satisfy attribute-value correspondence ((C:IBM, $\overline{\mathrm{D}: \text { stkCode }}),(\overline{\mathrm{C}: \mathrm{IBM}}, \overline{\mathrm{D}: \text { clsPrice }}))$, since "IBM" occurs as the name of attribute "C:IBM" and as the value of attribute "D:stkCode", and "196" occurs as the value of both attributes "C:IBM" and "D:clsPrice".

- Relation-attribute correspondence $((A, b),(\bar{a}, \bar{b}))$

$s$ and $t$ satisfy correspondence $((A, b),(\bar{a}, \bar{b}))$, if $A=b$ and $s^{a}=t^{b}$.

Example: The first tuple of database $\mathrm{R}$ and the first tuple of database $\mathrm{C}$ satisfy relation-attribute correspondence ((R:IBM, C:IBM), ( $\overline{\mathrm{R}: c l s P r i c e}, \overline{\mathrm{C}: \mathrm{IBM}}))$, because "IBM" occurs as the name of relation "R:IBM" and as the name of attribute "C:IBM", and "196" occurs as the value of both attributes "R:clsPrice" and "C:IBM".

- Relation-value correspondence $\left(\left(A, \overline{b_{1}}\right),\left(\bar{a}, \overline{b_{2}}\right)\right)$

$s$ and $t$ satisfy correspondence $\left(\left(A, \overline{b_{1}}\right),\left(\bar{a}, \overline{b_{2}}\right)\right)$, if $A=$ $t^{b_{1}}$ and $s^{a}=t^{b_{2}}$.

Example: The first tuple of database $\mathrm{R}$ and the second tuple of D satisfy relation-value correspondence ((R:IBM, $\overline{\mathrm{D}: \text { stkCode }}),(\overline{\mathrm{R}: c l s P r i c e}, \overline{\mathrm{D}: \text { clsPrice }}))$, because "IBM" occurs as the name of relation "R:IBM" and as the value of attribute "D:stkCode", and "196" occurs as the value of both attributes "R:clsPrice" and "D:clsPrice".

The semantics of attribute-value and relation-value correspondences involve two data values in tuple $t$ at the same time. These semantics show the dependency between the two primitive correspondences in a compound correspondence.

Given the definition of correspondences, a schema matching is a set of relation matchings, each of which includes a set of correspondences.

Definition 2.4 (RElation matching). Given two relations $A$ and $B$, a relation matching $M$ is a triple $(A, B, \Sigma)$, where $\Sigma$ is a set of correspondences.

Definition 2.5 (SCHEMA MATCHing). Given two schemas $\mathcal{A}$ and $\mathcal{B}$, a schema matching $\mathcal{M}$ is a set of relation matchings, each of which is between a relation of $\mathcal{A}$ and a relation of $\mathcal{B}$.

\section{GENERATING CORRESPONDENCES}

Because of dependencies, automatically generating compound correspondences is more difficult than generating the conventional attribute-attribute correspondences. For generating attribute-attribute correspondences, instance-based matchers need only determine if two attributes have similar values. For compound correspondences, the dependencies may not be evident for all values of an attribute. For example, to generate ((C:IBM, $\overline{\mathrm{D}: \mathrm{stkCode}}),(\overline{\mathrm{C}: \mathrm{IBM}}, \overline{\mathrm{D}: \mathrm{clsPrice}}))$, a matcher need compare the values of attribute "C:IBM" and the values of "D:clsPrice" when the values of "D:stdCode" is "IBM" for the same tuples.

Two algorithms are proposed to automatically generate correspondences. The first algorithm relies on the existence of duplicates in two databases. The second is a general instance-based method. Both methods require basic similarity measures. $S_{\text {field }}$ is a measure of similarity between two database elements. $S_{\text {list }}$ measures the similarity between two lists of elements. Similarity measures have been well studied [7]. Any similarity measure can be used.

\subsection{Duplicate Method}

The DUMAS system demonstrates that schema matching using duplicates is effective, and only needs a small number of duplicate pairs to achieve reasonable accuracy [5]. The basic idea is to first find similar tuples, and determine schema matchings based on the similarity of values in those tuples.

Our matching problem considers different types of database elements. We extend the DUMAS system in two ways to solve our problem. First, define a schema tuple as a uniform representation of both schema information and data values in a tuple. Record linkage algorithms over schema tuples can align any type of database elements. Second, the algorithms for generating correspondences based on duplicates are different because of the dependencies in compound correspondences.

The first subproblem of all duplicate-based methods is to find duplicates through record linkage. The record linkage algorithm in DUMAS computes similarity between two tuples as sets of strings. We apply a similar algorithm to 
schema tuples, which includes schema information in each tuple.

Definition 3.1 (SChema tuple). Given a relation $A$ and a tuple $t$, a schema tuple $\hat{t}$ is a set such that $\hat{t}=$ $\{A\} \cup\left\{a_{i} \mid a_{i} \in A\right.$, for $\left.i=1, \ldots,|A|\right\} \cup t$, where $A$ is interchangeably used to represent the name of relation $A$ and the set of all attributes in $A$, and $a_{i}$ is an attribute of $A$.

In database $\mathrm{D}$, the schema tuple of the first tuple is a set \{Stock, date, stkCode, clsPrice, 1/2/13, AAPL, 549\}.

Given two relations $A$ and $B$, the algorithm computes similarity between each pair of schema tuples. Each pair of schema tuples are tokenized into a set of terms. TFIDF is used for weighting terms. Specifically, the weight of a term $\tau$ in a schema tuple $\hat{t}$ is computed as:

$$
w^{\prime}(\hat{t}, \tau)=\log \left(t f_{\hat{t}, \tau}+1\right) \cdot \log \left(\frac{n}{d f_{\tau}}+1\right)
$$

where $t f_{\hat{t}, \tau}$ is the term frequency of $\tau$ in $\hat{t}, d f_{\tau}$ is the number of schema tuples in which $\tau$ appears, and $n$ is the total number of schema tuples. These weights are normalized for each schema tuple as:

$$
w(\hat{t}, \tau)=\frac{w^{\prime}(\hat{t}, \tau)}{\sqrt{\sum_{\tau^{\prime} \in \hat{t}} w^{\prime}\left(\hat{t}, \tau^{\prime}\right)^{2}}}
$$

where $\tau \in \hat{t}$ means $\tau$ is a term of $\hat{t}$ after tokenization. The similarity between two schema tuples $\hat{t}_{1}$ and $\hat{t}_{2}$ are computed as:

$$
S_{\text {tuple }}\left(\hat{t}_{1}, \hat{t}_{2}\right)=\sum_{\tau \in \hat{t}_{1} \cap \hat{t}_{2}} w\left(\hat{t}_{1}, \tau\right) \cdot w\left(\hat{t}_{2}, \tau\right)
$$

These pairs of schema tuples are ranked by the similarity in descending order. Given a parameter $N$, the top $N$ pairs of schema tuples are predicted as duplicates.

The next subproblem is to measure the confidence of every correspondence given the $N$ duplicate pairs. For each correspondence, the idea is to compute a score to measure how likely a duplicate pair satisfy the correspondence based on the similarity measure $S_{\text {field }}$. The confidence measure of the correspondence is the average of the scores across all duplicate pairs. All correspondences above a confidence threshold are considered as predicted correspondences.

Given two relations $A$ and $B$, denote the attributes $a \in$ $A$, and $b, b_{1}, b_{2} \in B$. The tuple of $A$ in the $i$ th duplicate pairs is denoted $I_{i}$, and the tuple of $B$ in the $i$ th duplicate pairs is denoted $J_{i}$. The confidence of correspondence $\sigma$ is represented as $p(\sigma)$.

\section{Attribute-attribute correspondence $(a, b)$.}

$$
p((a, b))=\frac{\sum_{i} S_{\text {field }}\left(I_{i}^{a}, J_{i}^{b}\right)}{N}
$$

\section{Attribute-value correspondence $\left(\left(a, \overline{b_{1}}\right),\left(\bar{a}, \overline{b_{2}}\right)\right)$.}

The attribute-value correspondences involve data values. Thus, we need to distinguish individual data values. The $N$ duplicate pairs are grouped by the unique data values of attribute $b_{1}$. A score is computed for each group, and the maximum score over all groups is the confidence of the correspondence.

$$
\begin{aligned}
& p\left(\left(\left(a, \overline{b_{1}}\right),\left(\bar{a}, \overline{b_{2}}\right)\right)\right) \\
& \quad=\max _{v \in J^{b_{1}}} \frac{\sum_{i, J_{i}^{b_{1}}=v} \sqrt{S_{\text {field }}(a, v) \cdot S_{\text {field }}\left(I_{i}^{a}, J_{i}^{b_{2}}\right)}}{\sum_{i, J_{i}^{b_{1}}=v} 1}
\end{aligned}
$$

where $J^{b_{1}}$ represents the set of unique values of attribute $b_{1}$ in all duplicate tuples.

\section{Relation-attribute correspondence $((A, b),(\bar{a}, \bar{b}))$.}

$$
p(((A, b),(\bar{a}, \bar{b})))=\frac{\sum_{i} \sqrt{S_{\text {field }}(A, b) \cdot S_{\text {field }}\left(I_{i}^{a}, J_{i}^{b}\right)}}{N}
$$

\section{Relation-value correspondence $\left(\left(A, \overline{b_{1}}\right),\left(\bar{a}, \overline{b_{2}}\right)\right)$.}

The relation-value correspondences also need to distinguish individual data values.

$$
\begin{aligned}
& p\left(\left(\left(A, \overline{b_{1}}\right),\left(\bar{a}, \overline{b_{2}}\right)\right)\right) \\
& \quad=\max _{v \in J^{b_{1}}} \frac{\sum_{i, J_{i}^{b_{1}}=v} \sqrt{S_{\text {field }}(A, v) \cdot S_{\text {field }}\left(I_{i}^{a}, J_{i}^{b_{2}}\right)}}{\sum_{i, J_{i}^{b_{1}}=v} 1}
\end{aligned}
$$

\subsection{Non-duplicate Method}

The non-duplicate method is a general-purpose solution, which does not require duplicate data. As long as the two databases have data, the non-duplicate method can be used. As in the duplicate method, the task is to measure the confidence of every correspondence. All correspondences with confidence above a threshold are considered as predicted correspondences.

Instead of computing the average similarity of all duplicate pairs in the duplicate method, the non-duplicate method estimates the confidence using the similarity measure between lists of data values, $S_{\text {list }}$.

The list of all values of attribute $a$ in relation $A$ is represented as $I^{a}$, and the list of all values of attribute $b$ in relation $B$ is represented as $J^{b}$.

\section{Attribute-attribute correspondence $(a, b)$.}

$$
p((a, b))=S_{\text {list }}\left(I^{a}, J^{b}\right)
$$

\section{Attribute-value correspondence $\left(\left(a, \overline{b_{1}}\right),\left(\bar{a}, \overline{b_{2}}\right)\right)$.}

Similar to the duplicate method, the tuples are grouped by the unique values of attribute $b_{1}$. A score is computed for each group, and the maximum score is the confidence of the correspondence.

$$
p\left(\left(\left(a, \overline{b_{1}}\right),\left(\bar{a}, \overline{b_{2}}\right)\right)\right)=\max _{v \in J^{b_{1}}} \sqrt{S_{\text {field }}(a, v) \cdot S_{\text {list }}\left(I^{a}, J_{v}^{b_{2}}\right)}
$$

where $J_{v}^{b_{2}}$ represents the list of all values of attribute $b_{2}$ in the tuples having the value of attribute $b_{1}$ as $v$.

3. Relation-attribute correspondence $((A, b),(\bar{a}, \bar{b}))$.

$$
p(((A, b),(\bar{a}, \bar{b})))=\sqrt{S_{\text {field }}(A, b) \cdot S_{\text {list }}\left(I^{a}, J^{b}\right)}
$$




\section{Relation-value correspondence $\left(\left(A, \overline{b_{1}}\right),\left(\bar{a}, \overline{b_{2}}\right)\right)$.}

The tuples are also grouped by the unique values of attribute $b_{1}$, and the confidence is computed as the maximum score of all groups.

$$
p\left(\left(\left(A, \overline{b_{1}}\right),\left(\bar{a}, \overline{b_{2}}\right)\right)\right)=\max _{v \in J^{b_{1}}} \sqrt{S_{\text {field }}(A, v) \cdot S_{\text {list }}\left(I^{a}, J_{v}^{b_{2}}\right)}
$$

\subsection{Implementation}

Field similarity, $S_{\text {field }}$, measures the similarity between two database elements. Any similarity measure can be used. For example, DUMAS uses SoftTFIDF [7]. SoftTFIDF takes into account similar terms, while conventional TFIDF only considers equal terms. We implement a similar measure as DUMAS with two differences. First, because IDF is not well defined in our problem, we use SoftTF instead of SoftTFIDF. Second, we make the measure as symmetric by averaging the two directions of DUMAS's asymmetric measure.

The list similarity, $S_{\text {list }}$, measures the similarity between two lists of database elements. We use statistics of the two lists to measure similarity. Since our problem does not have human labels, the supervised machine learning algorithms cannot be used in our problem.

Our measure of list similarity is specialized for different data types. The current implementation details three data types: string, number, and date. Given a list, all elements are attempted to be parsed to the three types. The type with the most successfully parsed elements is the estimated data type for the list. The statistics are extracted as a vector from all elements that can be parsed as the estimated data type. For string, the vector contains term frequencies (TF) after tokenizing all elements. For number, a 20-bin histogram is generated to represent the distribution of all elements. Before generating the histogram, all elements are sorted, and only the middle $95 \%$ are used to eliminate outliers. For date, strings such as "January" and "Monday" are converted to numbers, and the vector contains the frequencies of numbers with one to four digits. Note that if an element has more than three numbers (a date at most contains year, month, and day) or less than two numbers (should be a number instead of date) or more than one number with three or four digits (only year has three or four digits), that element is not parsed as a date. The similarity between two lists $l_{1}$ and $l_{2}$ that has the same estimated data type is computed as follows:

$$
S_{\text {list }}\left(l_{1}, l_{2}\right)=\sqrt{S_{\text {type }}\left(l_{1}, l_{2}\right) \cdot S_{\text {stat }}\left(l_{1}, l_{2}\right)}
$$

where $S_{\text {type }}\left(l_{1}, l_{2}\right)$ is the minimum of percentage of the elements that are parsed as the estimated data type in $l_{1}$ and $l_{2}$. $S_{\text {stat }}\left(l_{1}, l_{2}\right)$ is cosine similarity between the two vectors of statistics.

When predicting attribute-value and relation-value correspondences, the value $v$ in (2) and (4) are required to occur in at least two, and at least $10 \%$ duplicate pairs to eliminate outliers. The value $v$ in (6) and (8) are required to occur in at least $10 \%$ tuples. In addition, the value $v$ is unlikely to occur in the attributes with many unique values. We prune the attributes with more than 10 unique values to improve speed. If a predicted attribute-attribute correspondence has lower confidence than its corresponding compound correspondences, the attribute-attribute correspondence will be ignored.

\section{TUPLE-GENERATING DEPENDENCY}

In this section, we introduce the algorithm to formulate compound correspondences as tgds with constants. We also discuss how to use these tgds in data exchange and data integration applications.

\subsection{Formulation}

Given a target schema $\mathcal{A}$ and a source schema $\mathcal{B}$, a tuplegenerating dependency is a first-order logic formula in the form:

$$
\forall \mathbf{x}(\phi(\mathbf{x}) \rightarrow \exists \mathbf{y} \psi(\mathbf{x}, \mathbf{y}))
$$

where $\phi(\mathbf{x})$ is a conjunction of atomic formulas over $\mathcal{B}$, and $\psi(\mathbf{x}, \mathbf{y})$ is a conjunction of atomic formulas over $\mathcal{A}$.

With the conventional schema matchings that only contain attribute-attribute correspondences, a tgd assigns variables to the attributes of $\mathcal{A}$ and $\mathcal{B}$. The matched attributes share the same variable.

Compound correspondences defined in this paper involve matchings of relations, attributes, and data values. Representations of compound correspondences need higher-order logic as shown by Krishnamurthy, Litwin, and Kent, which cannot be expressed as tgds [18]. We observe that relations and attributes are rarely changed, and if they are changed, the correspondences have to be re-computed. Thus, we can consider relations and attributes as constants in respect to correspondences. Instead of representing compound correspondences as higher-order logic, we represent them in firstorder logic (tgds) by substituting relations and attributes with their names as constants.

For example, ((C:IBM, $\overline{\mathrm{D}: \text { stkCode }}),(\overline{\mathrm{C}: \mathrm{IBM}}, \overline{\mathrm{D}: \mathrm{clsPrice}}))$ can be represented in tgd as:

$$
\begin{gathered}
\forall x_{1} x_{2} x_{3} x_{4}\left(\mathrm{C}: \operatorname{Stock}\left(x_{1}, x_{2}, x_{3}, x_{4}\right) \rightarrow\right. \\
\left.\exists y \text { D:Stock }\left(y, " I B M ", x_{3}\right)\right)
\end{gathered}
$$

where "IBM" is a constant.

\subsection{Generation}

For simplicity, we generate tgds from one relation of the target schema $\mathcal{A}$ to one relation of the source schema $\mathcal{B}$. The generation of tgds including more than one relation is discussed later. Given relations $A \in \mathcal{A}, B \in \mathcal{B}$, and a set of correspondences $\Sigma$ between $A$ and $B$, the task is to formulate $\Sigma$ as a set of tgds.

The preliminary step of formulating tgds is to group the correspondences in $\Sigma$ into subsets such that the correspondences in each subset are non-overlap. A tgd will be generated for each subset. This is important for compound correspondences, since multiple correct correspondences may share elements. ((C:IBM, $\overline{\mathrm{D}: \text { stkCode }}),(\overline{\mathrm{C}: \mathrm{IBM}}, \overline{\mathrm{D}: \mathrm{clsP} \text { rice }}))$ and $((\mathrm{C}: \mathrm{AAPL}, \overline{\mathrm{D}: \mathrm{stkCode}}),(\overline{\mathrm{C}: \mathrm{AAPL}}, \overline{\mathrm{D}: c l s P r i c e}))$ are examples.

We first define the overlap of two correspondences. Correspondences can contain relations, attributes, and symbols representing data values. The overlap includes the common elements in the two correspondences. In addition, we are also concerned with the attributes underlying the symbols representing data values.

DEFINITION 4.1 (NON-OVERLAP CORRESPONDENCE). Given two correspondences $\sigma_{1}$ and $\sigma_{2}, \Theta_{1}$ and $\Theta_{2}$ are the sets containing all database elements in $\sigma_{1}$ and $\sigma_{2}$, respectively. $\sigma_{1}$ and $\sigma_{2}$ are non-overlap, if: 


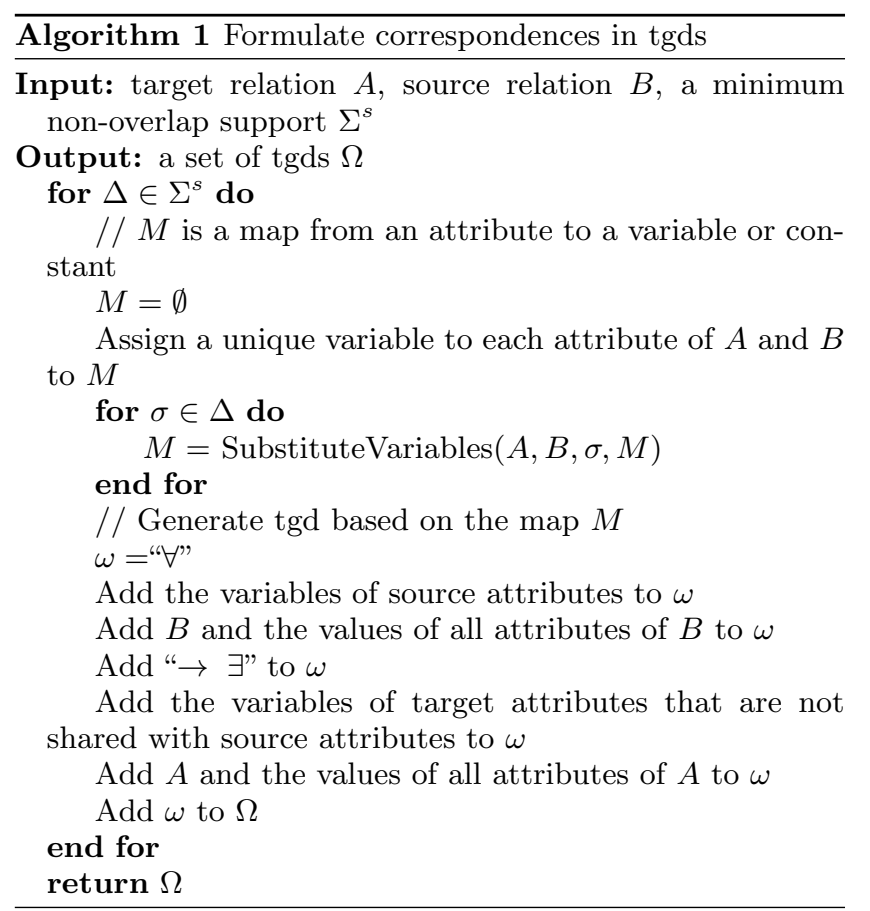

- $\Theta_{1} \cap \Theta_{2}=\emptyset$

- Denote $\left\{a_{0}, \ldots, a_{i}\right\}$ and $\left\{\overline{b_{0}}, \ldots, \overline{b_{j}}\right\}$ as the set of attributes and the set of symbols representing data values in $\Theta_{1}$, and $\left\{c_{0}, \ldots, c_{k}\right\}$ and $\left\{\overline{d_{0}}, \ldots, \overline{d_{l}}\right\}$ as the set of attributes and the set of symbols representing data values in $\Theta_{2} .\left\{a_{0}, \ldots, a_{i}\right\} \cap\left\{d_{0}, \ldots, d_{l}\right\}=\emptyset$ and $\left\{b_{0}, \ldots, b_{j}\right\} \cap\left\{c_{0}, \ldots, c_{k}\right\}=\emptyset$.

DEFINITION 4.2 (NON-OVERLAP CORRESPONDENCE SET). $A$ set of correspondences $\Sigma$ is non-overlap, if $\forall \sigma_{1}, \sigma_{2} \in \Sigma$ that $\sigma_{1} \neq \sigma_{2}, \sigma_{1}$ and $\sigma_{2}$ are non-overlap.

Given a set of correspondences $\Sigma$, a minimum non-overlap support $\Sigma^{s}$ is defined as a set of subsets of $\Sigma$, each of which is a non-overlap correspondence set and is as large as possible.

DEFINITION 4.3 (MINIMUM NON-OVERLAP SUPPORT). Given a set of correspondences $\Sigma$, a set $\Sigma^{s}$ is a minimum non-overlap support of $\Sigma$ if the following conditions are satisfied:

- $\forall \Delta \in \Sigma^{s}, \Delta \subseteq \Sigma$;

- $\forall \Delta \in \Sigma^{s}, \Delta$ is a non-overlap correspondence set;

- $\forall \Delta^{\prime} \subseteq \Sigma$ that is non-overlap, $\exists \Delta \in \Sigma^{s}$ such that $\Delta^{\prime} \subseteq$ $\Delta$;

- $\forall \Delta_{1}, \Delta_{2} \in \Sigma^{s}, \Delta_{1} \neq \Delta_{2}$, then $\Delta_{1} \not \subset \Delta_{2}$ and $\Delta_{2} \not \subset$ $\Delta_{1}$.

Given the fact that the number of correspondences is usually small, the minimum non-overlap support can be found by brute force.

Algorithm 1 and 2 describe the details of generating tgds given a minimum non-overlap support. The algorithms maintain a map data structure with all attributes of both $A$ and $B$ as keys, and strings as values. The map is initialized

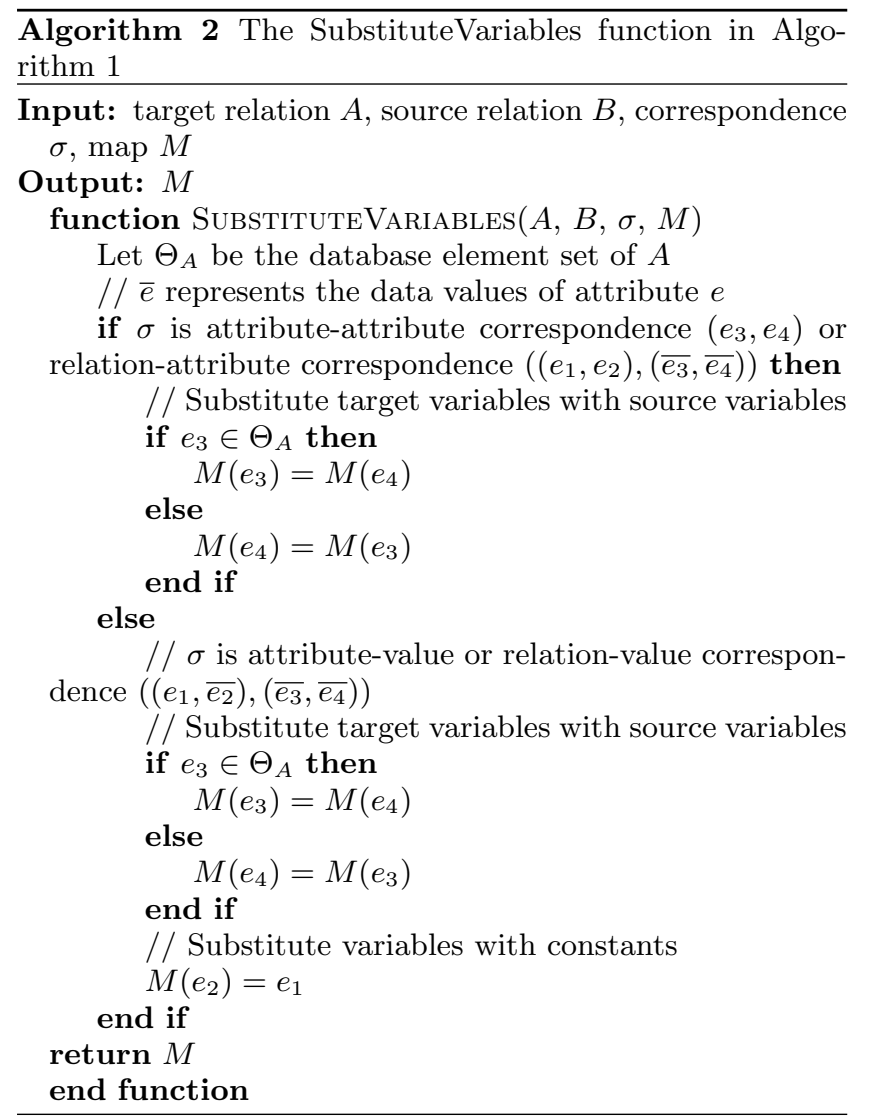

by assigning a unique variable to each attribute. The algorithms visit each correspondence, and substitute some variables in the map with other variables or constants according to the correspondence. Finally, a logic representation is generated based on the map. The variable substitutions for a relation-attribute correspondence $\left(\left(e_{1}, e_{2}\right),\left(\overline{e_{3}}, \overline{e_{4}}\right)\right)$ and a corresponding attribute-attribute correspondence $\left(e_{3}, e_{4}\right)$ are the same. This is due to the fact that the primitive correspondence $\left(e_{1}, e_{2}\right)$ is between a relation and an attribute. Both relation and attribute are constants, so there is no variable to substitute. This fact shows that a relation-attribute correspondence does not provide more information than its corresponding attribute-attribute correspondence in a tgd. For attribute-value and relation-value correspondences, the substitution involves constants. Both variables of the target attributes and the source attributes may be substituted with constants.

In the examples in Figure 2, let databases $\mathrm{D}$ and $\mathrm{C}$ be the target and source databases, respectively. The set of correspondences $\Sigma$ is:

$$
\begin{aligned}
\Sigma=\{ & (\text { C:date }, \text { D:date }), \\
& ((\text { C:AAPL }, \overline{\mathrm{D}: \text { stkCode }}),(\overline{\mathrm{C}: \mathrm{AAPL}}, \overline{\mathrm{D}: \text { clsPrice }})), \\
& ((\mathrm{C}: \mathrm{IBM}, \overline{\mathrm{D}: \mathrm{stkCode}}),(\overline{\mathrm{C}: \mathrm{IBM}}, \overline{\mathrm{D}: \mathrm{clsPrice}}))\}
\end{aligned}
$$




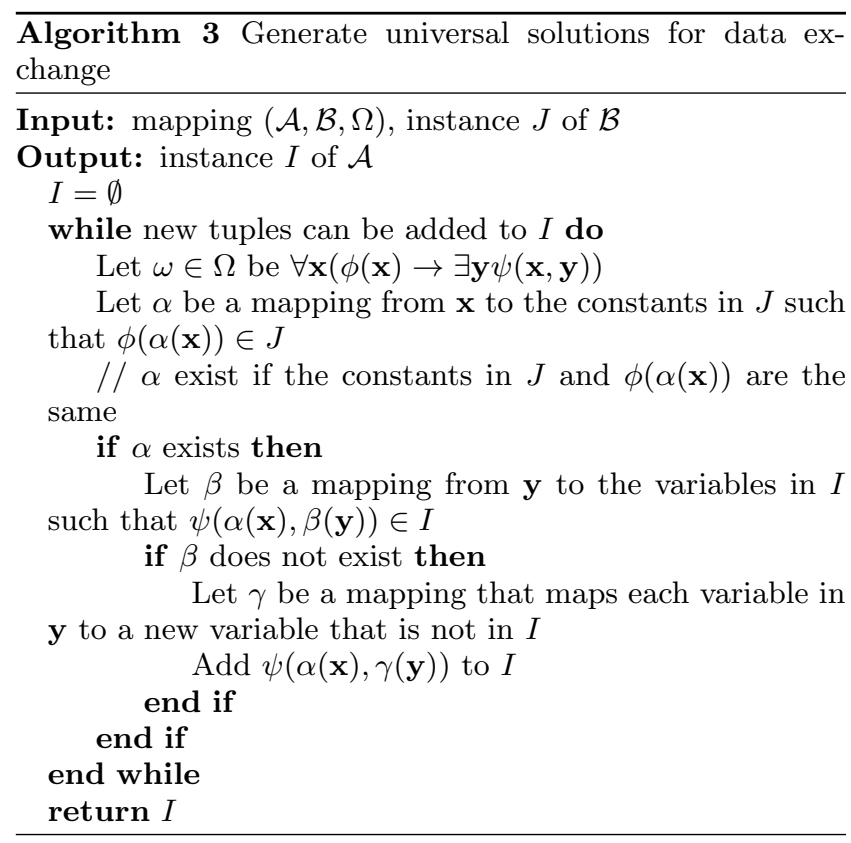

The set of generated tgds is:

$$
\begin{gathered}
\Omega=\left\{\forall x _ { 1 } x _ { 2 } x _ { 3 } x _ { 4 } \left(\mathrm{C}: \operatorname{Stock}\left(x_{1}, x_{2}, x_{3}, x_{4}\right) \rightarrow\right.\right. \\
\text { D:Stock } \left.\left(x_{1}, " \operatorname{AAPL} ", x_{2}\right)\right), \\
\forall x_{1} x_{2} x_{3} x_{4}\left(\mathrm{C}: \operatorname{Stock}\left(x_{1}, x_{2}, x_{3}, x_{4}\right) \rightarrow\right. \\
\left.\left.\operatorname{D}: \operatorname{Stock}\left(x_{1}, " \operatorname{IBM} ", x_{3}\right)\right)\right\}
\end{gathered}
$$

\section{tgds with multiple relations.}

The problem of generating schema mappings with multiple relations has been discussed in Clio, which semi-automatically generates mappings as tgds [14]. The mappings are created in two steps: finding initial correspondences between attributes and associating correspondences by logical inference through referential constraints. The correspondence association is done by a modification of the chase algorithm [20]. To generate schema mappings with multiple relations in our problem, we can directly apply the method of correspondence association in Clio to the tgds between a pair of relations generated by Algorithm 1.

\subsection{Applications}

\section{Data Exchange.}

Data exchange is the problem of transforming an instance of a source schema to an instance of the target schema, given a mapping between the two schemas $[15,1]$. Let us denote the target schema as $\mathcal{A}$ and the source schema as $\mathcal{B}$. A mapping, $\mathcal{M}$, is defined as a triple $(\mathcal{A}, \mathcal{B}, \Omega)$, where $\Omega$ is a set of source-to-target dependencies. In this definition, we ignore the constraints on the target schema, and only consider the source-to-target dependencies. The source-totarget dependencies are usually tgds.

A target instance $I$ is a solution of a source instance $J$ under mapping $\mathcal{M}$, if $I$ and $J$ satisfy $\Omega$. There may exist more than one solution of a source instance. A universal solution is a target instance that contains no more and no less than what the mapping specification requires. As it is widely accepted in the data exchange literature, universal solutions reflect the source data better [1].

Generating universal solutions is an important task in data exchange. The chase algorithm is generally used to find universal solutions $[20,15]$. Given a source instance, the chase algorithm iteratively visits a tgd in the schema mapping, and generates a target tuple. The process is stopped until no new target tuples can be generated.

In our problem, tgds include constants to represent compound correspondences. We extend the chase algorithm to generate universal solutions for our problem. Algorithm 3 is a slightly modified chase algorithm based on the version in [10]. The only change in Algorithm 3 is the check of whether the constants in the source instance and the tgds are the same.

Given the tgds in (10) and the instance of database C in Figure 2, the universal solutions are:

$$
\begin{aligned}
& (1 / 2 / 13, \text { AAPL }, 549) \\
& (1 / 2 / 13, \text { IBM, } 196) \\
& (1 / 3 / 13, \text { AAPL }, 542) \\
& (1 / 3 / 13, \text { IBM, } 195)
\end{aligned}
$$

\section{Data Integration.}

Data integration provides uniform access to a set of autonomous, heterogeneous structured data sources [10]. Given a target schema $\mathcal{A}$, a set of source schemas $\mathcal{B}_{1}, \mathcal{B}_{2}, \ldots$, schema mappings $\Omega$, and a user query $q_{\mathcal{A}}$ over $\mathcal{A}$, the task of data integration is to rewrite $q_{\mathcal{A}}$ to queries over the source schemas and answer $q_{\mathcal{A}}$ using the data in the data sources. The schema mappings can be represented in three formalisms. Global-as-view (GAV) represents each relation in the target schema as a view of the source schemas. Local-as-view (LAV) represents each relation in the source schemas as a view of the target schema. The combination of GAV and LAV is Global-local-as-view (GLAV), which includes a mapping from a view of the target schema to a view of the source schemas.

Query rewriting is an important task in data integration. The rewriting in GAV formalism is simply query unfolding. The rewriting in LAV formalism is more complex. It needs algorithms for answering queries using views, such as MiniCon [21]. The rewriting in GLAV formalism combines the rewriting in both GAV and LAV. The algorithm contains two steps: (1) rewrite the target query using the views of the target schema in the schema mappings, (2) replace the views of the target schema by the views of the source schema and unfold the queries.

In our problem, the schema mappings are represented as tgds, which are equivalent in expressiveness to GLAV formalism. Thus, the rewriting algorithms for GLAV can be applied to tgds. MiniCon is a well-known rewriting algorithm that translates a conjunctive query to union of conjunctive queries [21]. It supports constants in both queries and mappings. Thus, the MiniCon algorithm can be directly applied to the tgds in our solution.

In the examples in Figure 2, let databases $\mathrm{D}$ and $\mathrm{C}$ be the target and source databases, respectively. The schema mappings are given in (10). Consider a query $Q(x, y)$ over database D asking the prices of all stocks of date " $1 / 2 / 13$ ". For clarity, queries are written in relational calculus.

$$
Q(x, y):-\mathrm{D}: \operatorname{Stock}(1 / 2 / 13, x, y)
$$


The first step of the rewriting represents the query using union of two conjunctive views of database $\mathrm{D}$ in the tgds:

$$
\begin{aligned}
& Q^{\prime}(x, y):-\mathrm{D}: \operatorname{Stock}(1 / 2 / 13, \mathrm{AAPL}, y), x=\mathrm{AAPL} \\
& Q^{\prime}(x, y):-\mathrm{D}: \operatorname{Stock}(1 / 2 / 13, \mathrm{IBM}, y), x=\mathrm{IBM}
\end{aligned}
$$

The final rewritten query is union of two conjunctive views of the source schema $\mathrm{C}$ :

$$
\begin{aligned}
& Q^{\prime \prime}(x, y):-\mathrm{C}: \operatorname{Stock}\left(1 / 2 / 13, y, z_{1}, z_{2}\right), x=\mathrm{AAPL} \\
& Q^{\prime \prime}(x, y):-\mathrm{C}: \operatorname{Stock}\left(1 / 2 / 13, z_{3}, y, z_{4}\right), x=\mathrm{IBM}
\end{aligned}
$$

Comparing $Q(x, y)$ and $Q^{\prime \prime}(x, y)$, the target query is a conjunctive query, but the rewritten query is a union of two conjunctive queries. Recall that the schema mappings between database $\mathrm{C}$ and $\mathrm{D}$ include attribute-value correspondences. Although we represent the attribute-value correspondences in first-order logic, the semantics still need second-order logic. Thus, the rewritten query needs to enumerate (union) the attributes to express the second-order logic by first-order logic.

\section{EXPERIMENTS}

\subsection{Data sets}

The data sets comprise four application domains: Ecommerce, Stock, Enrollment, and Game.

The Ecommerce data set contains two schemas that are in commercial use. The first one is from the backend database of an open source ecommerce software ${ }^{1}$, populated with used car listings from a car dealer website $e^{2}$. The second one is from the backend database of an auto classified software ${ }^{3}$ that includes demo data. This schema contains 39 attributes in a single relation describing car information. The integer foreign keys are substituted with real values if each key refers to a unique value. Figure 4 shows the two ecommerce schemas.

The Stock data set contains real stock prices of three IT companies (AAPL, GOOG, and IBM) in 2012, downloaded from Yahoo! Finance ${ }^{4}$. The data is transformed in the formats of the three real world schemas described in [18].

The Enrollment data set includes a schema of college enrollment statistics downloaded from the National Center for Education Statistics ${ }^{5}$, and two schemas of college enrollment rankings crawled from Wikipedia ${ }^{6}$. The statistics schema contains the enrollment number of 358 colleges from 1990 to 2010. One ranking schema contains the top 10 enrolled colleges in 2009. The other combines the top 10 enrolled colleges from 2008 to 2012.

The game data set includes a schema of the global sales of computer games crawled from $\mathrm{VGChartz}^{7}$, and a schema of game information queried from DBpedia ${ }^{8}$. The schema from VGChartz contains 20000 games, and the schema from DBpedia contains 48132 triples.

\footnotetext{
${ }^{1}$ Opencart, http://www.opencart.com/

${ }^{2}$ http://www.centraltxautos.com/

${ }^{3}$ http://www.subrion.com/product/autos.html

${ }^{4}$ http://finance.yahoo.com/

${ }^{5}$ http://nces.ed.gov/

${ }^{6}$ http://www.wikipedia.org/

${ }^{7}$ http://www.vgchartz.com/

${ }^{8}$ http://dbpedia.org/
}

product_attribute
\begin{tabular}{|c|c|c|c|}
\hline product_id & attribute_name & language_name & text \\
\hline 1 & exterior & English & silver \\
1 & interior & English & other \\
1 & engine & English & $3.5 \mathrm{~L}$ V6 MPI SOHC 24V \\
1 & fuel & English & gasoline \\
1 & mileage & English & 42953
\end{tabular}

(a) Open source ecommerce software

autos
\begin{tabular}{|c|c|c|c|c|c|c|}
\hline id & mileage & exterior_color & engine & transmission & fuel_type & $\ldots$ \\
\hline 4 & 240000 & Blue & 6 cylinders & Manual & Gasoline & \\
5 & 160000 & Black & 4 cylinders & Automatic & Gasoline & \\
6 & 100000 & Blue & 4 cylinders & Automatic & Gasoline, Gas & $\ldots$ \\
7 & 32000 & Grey & 4 cylinders & Semi-automatic & Gasoline & \\
9 & 150000 & Gold & 12 cylinders & Manual & Gasoline &
\end{tabular}

(b) Auto classified software

Figure 4: The two schemas in Ecommerce data set that are in commercial use. This data set demonstrates the existence of attribute-value correspondences in real applications.

\begin{tabular}{l|c|c|c|c}
\hline & Ecommerce & Stock & Enrollment & Game \\
\hline attribute-attribute & 1 & 2 & 2 & 1 \\
attribute-value & 5 & 3 & 3 & 3 \\
relation-attribute & 0 & 1 & 1 & 0 \\
relation-value & 0 & 2 & 4 & 0 \\
\hline total & 6 & 8 & 10 & 4 \\
\hline
\end{tabular}

Table 1: The number of unique groundtruth correspondences of different types.

For all data sets, we manually determine groundtruth correspondences. Table 1 shows the number of unique groundtruth correspondences of different types. The portion of attribute-attribute correspondences is at most $25 \%$ in any of the data sets. This demonstrates the importance of compound correspondences. The entire test suite will be available on our website.

\subsection{Baselines}

DUMAS is the schema matching system that is closely related to our proposed duplicate method [5]. We implement DUMAS baselines as follows. First, the record linkage algorithm in Section 3.1 is applied to generate duplicate tuples, instead of schema tuples. A similarity matrix of all pairs of attributes is computed as in (1). All pairs with similarity above the threshold are the predicted correspondences. These baselines are named as DUMAS_x, where $\mathrm{x}$ is the number of duplicates used for matching. In the experiments, $\mathrm{x}$ is set to 5 and 10 as in our proposed methods.

We also implement a general-purpose instance-based matching baseline to compare with our proposed non-duplicate method. The instance-based matching baseline extracts the data of each attribute as a set, and compute a similarity matrix of all pairs of attributes as in (5). All pairs with similarity above the threshold are the predicted correspondences. The instance-based baseline is named Instance. Instance uses at most 1000 tuples from each schema for matching.

The proposed duplicate methods are Dup_5 and Dup_10, which use 5 and 10 duplicates, respectively. The proposed non-duplicate method is NonDup. NonDup uses the same tuples as Instance baseline. The main difference between the proposed methods and the baselines is that the baselines only predict attribute-attribute correspondences. 


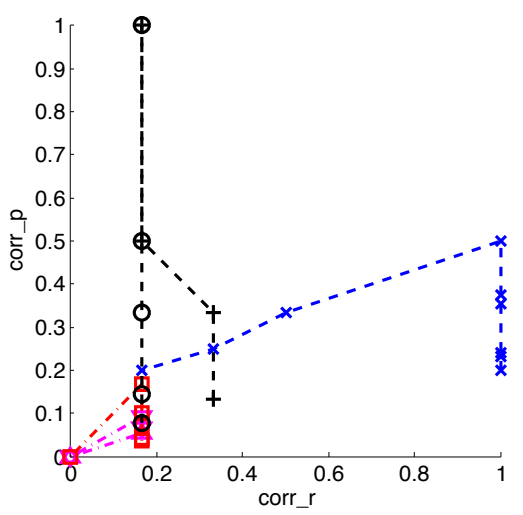

(a) Ecommerce

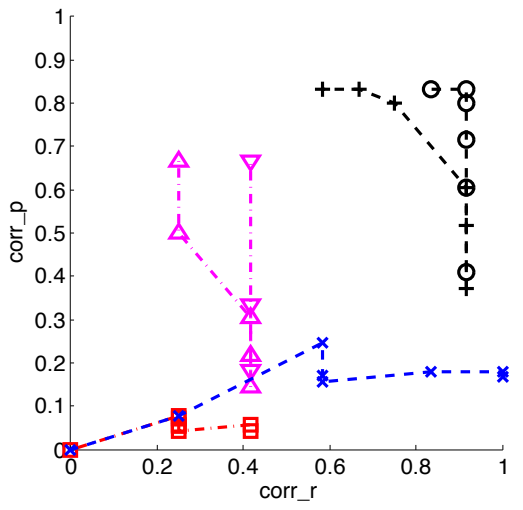

(c) Enrollment

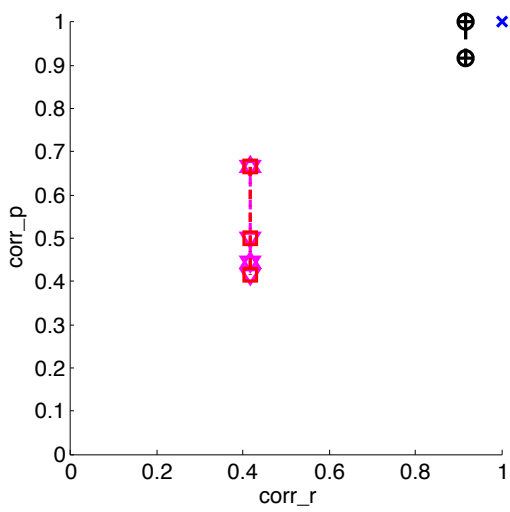

(b) Stock

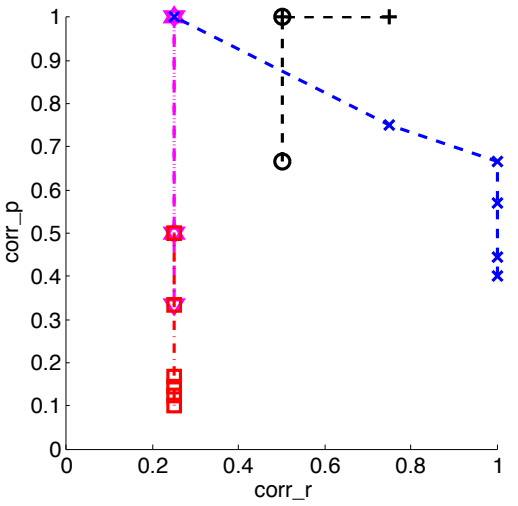

(d) Game

$-\nabla \cdot$ DUMAS_5 - $\triangle$ - DUMAS_10 - $\square \cdot$ Instance $-\Theta-$ Dup_5 - + - Dup_10 $-*-$ NonDup

(e) Legend

Figure 5: Precision-Recall curves obtained by varying correspondence threshold from 0.1 to 0.9 . Some points in the figures may be missing, due to the fact that no predicted correspondence has confidence higher than the threshold. One point may represent the results of multiple thresholds. Closer to the upper-right corner means better performance.

\subsection{Metrics}

We measure the precision, recall, and f-measure of correspondences. Denote the set of predicted correspondences as $P$, and the set of groundtruth correspondences as $T$. The precision (corr_p), recall (corr_r), and f-measure (corr_f) are defined as follows:

$$
\begin{aligned}
& \text { corr_p }(P, T)=\frac{|P| \cap|T|}{|P|}, \quad \text { corr_r }(P, T)=\frac{|P| \cap|T|}{|T|} \\
& \text { corr_f }(P, T)=2 \cdot \frac{\text { corr_p }(P, T) \cdot \text { corr_r }(P, T)}{\operatorname{corr} \_\mathrm{p}(P, T)+\text { corr_r }(P, T)}
\end{aligned}
$$

Per Section 4, a relation-attribute correspondence and its corresponding attribute-attribute correspondence are formulated to the same tgd. Thus, we do not distinguish a relationattribute correspondence and its corresponding attributeattribute correspondence in the evaluation.

Generating tgds based on correspondences is a deterministic process. Thus we do not measure the correctness of tgds in the experiments.

If a data set has more than two schemas, the experiments are conducted on each pair of schemas, and the average results are reported.

\subsection{Results}

The threshold to determine predicted correspondences has a large impact to all methods (predicted correspondences must have higher confidence than the threshold). We vary the threshold from 0.1 to 0.9 to give a comprehensive comparison.

A fair comparison should consider both precision and recall measures at the same time. Figure 5 shows the PrecisionRecall curves obtained by varying correspondence threshold. Precision-Recall curves are often used in information retrieval. These curves have recall as horizontal axis and precision as vertical axis. A good system should be close to the upper-right corner in the figure. Figure 6 shows the correspondence f-measures given various thresholds. F-measure is a tradeoff between precision and recall, and higher f-measure indicates better performance.

In summary, at least one of the proposed methods dominates all baselines in all four data sets. Comparing the duplicate methods with the non-duplicate method, the duplicate methods usually achieve higher precision while the non-duplicate method achieves higher recall. Their overall performance depends on individual data set.

The Ecommerce data set is challenging for all methods. 


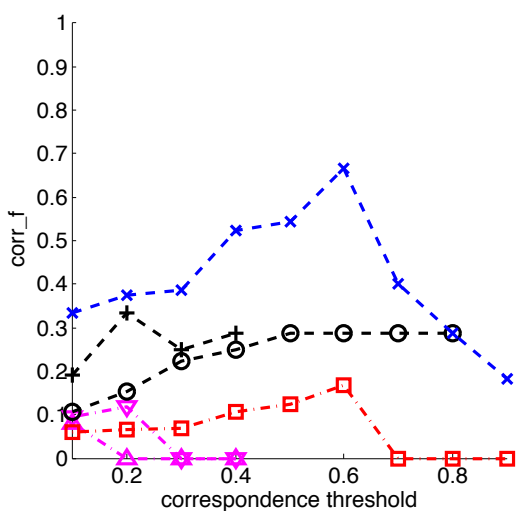

(a) Ecommerce

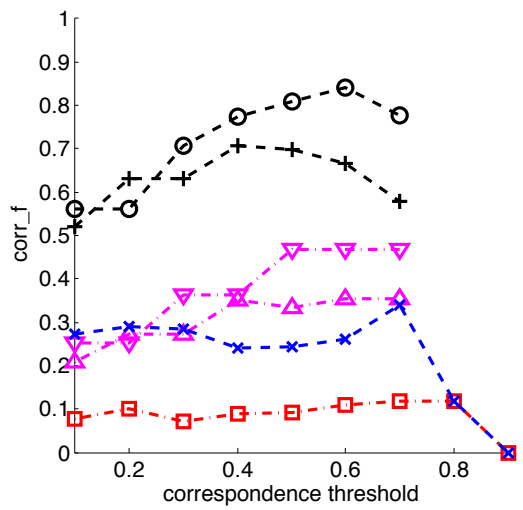

(c) Enrollment

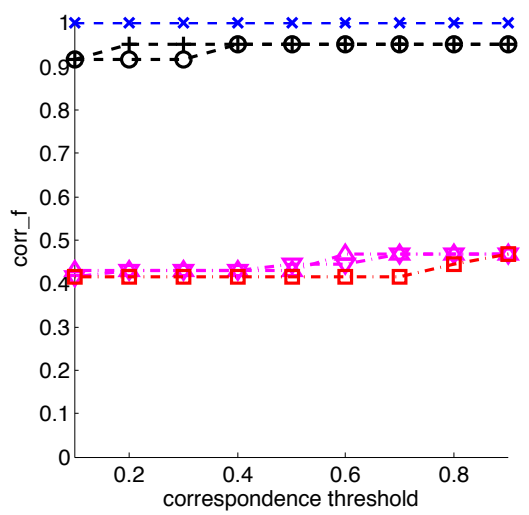

(b) Stock

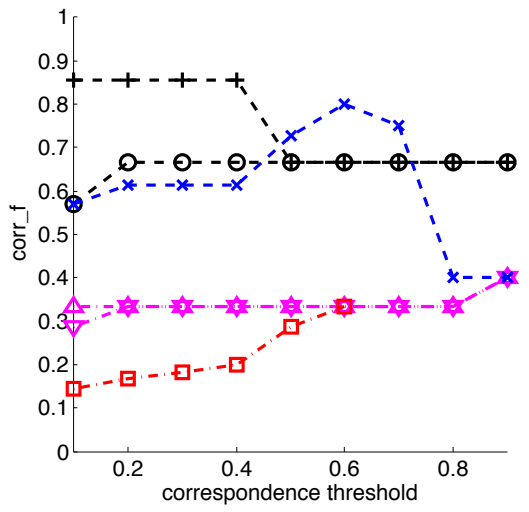

(d) Game

$-\nabla \cdot$ DUMAS_5 $-\triangle$ - DUMAS_10 - $\square \cdot$ Instance $-\Theta-$ Dup_5 -+- Dup_10 $-*-$ NonDup

(e) Legend

Figure 6: Correspondence f-measures (corr_f, vertical axis) with various correspondence thresholds (horizontal axis). Some points in the figures may be missing, due to the fact that no predicted correspondence has confidence higher than the threshold. Larger value means better performance.

First, the data of the two schemas are completely from independent data sources. There is no duplicate data. Second, one of the relations is large, containing 39 attributes. The data values of those attributes span many data types, including integer, real number, date, binary, url, and string. As shown in Figure 6, every proposed method performs better than all baselines. The top proposed method (NonDup with 0.6 threshold) has 0.667 of f-measure, while the top baseline (Instance with 0.6 threshold) only has 0.167 . NonDup dominates both Dup_5 and Dup_10 too. The NonDup achieves higher f-measure because of higher recall as shown in Figure 5 .

The data of all schemas in the Stock data set comes from the same source, so the comparison is mainly to highlight the importance of predicting all types of correspondences instead of only attribute-attribute. All proposed methods achieve f-measure above 0.9 , while all baselines are under 0.5 . The big gaps between the proposed methods and the baselines demonstrate the importance of predicting all types of correspondences.

For the Enrollment data set, Dup_5 and Dup_10 still dominate all baselines. NonDup performs better than or as well as Instance baseline, but worse than DUMAS_5 and
DUMAS_10. This is because most of the enrollment data are numbers of enrollment for different colleges in different years. The numbers are too similar to be distinguished by the non-duplicate methods. Dup_5 performs better than Dup_10 on the Enrollment data set. This is because the number of real duplicates in the data set is small. Thus, more duplicates introduce more errors to Dup_10.

Although the two schemas in the Game data set are from independent data sources, there exists duplicates as both data sources may share computer games. All proposed methods perform better than or as well as the top baseline. The Dup_5 and Dup_10 still dominate all baselines with big gaps. The NonDup method achieves the highest f-measure with threshold 0.5 to 0.7 , and the duplicate methods perform better for other thresholds.

The performance of the proposed duplicate methods is stable and robust on the Stock, Enrollment, and Game data sets, which contain some duplicates. Even on Enrollment data set with most of the data as numbers, the duplicate methods still achieve the highest performance. The drawback of these methods is the requirement of duplicate data from two schemas. However, our experimental results demonstrate that the number of required duplicate data is small. 
The top method only needs 5 duplicates. This small requirement enables duplicate methods to be used in broad applications.

The non-duplicate method is general for all schemas with data. On the Ecommerce, Stock and Game sets, NonDup performs better than the duplicate methods given certain correspondence thresholds. The performance is highly dependent on the capabilities of the underlying similarity measures. On Enrollment data set, NonDup is dominated by Dup_5 and Dup_10 with large gaps, because the similarity measures are confused by the numeric data.

The proposed duplicate methods usually achieve higher precision, while the proposed non-duplicate method achieves higher recall. This is illustrated by the Ecommerce and Game data sets in Figure 5. For the Ecommerce data set, the duplicate methods can have as high as 1.0 of precision, but lower than 0.4 of recall. The non-duplicate method can have 1.0 of recall, but lower than 0.5 of precision. This indicates that the duplicate and non-duplicate methods should be used in applications with different requirements. The duplicate methods give high accuracy, while the non-duplicate method gives high coverage.

Correspondence threshold has a large impact on performance. Higher threshold gives higher precision, but lower recall. The optimal choice of threshold is dependent on data sets. For the schemas with higher data similarity, such as the Stock data set, a higher threshold should be chosen. On the contrary, a lower threshold gives better performance for the schemas with lower data similarity, such as the Game data set.

\subsection{Error Categorization}

To detail insight into the proposed methods, we categorize the main problems observed in the experiments.

1. Attribute-value and relation-value correspondences have similar confidence to their corresponding attribute-attribute correspondences. By definition, the elements of an attributevalue correspondence $\left(\left(a, \overline{b_{1}}\right),\left(\bar{a}, \overline{b_{2}}\right)\right)$ form two primitive correspondences $\left(a, \overline{b_{1}}\right)$ and $\left(\bar{a}, \overline{b_{2}}\right)$. The elements in an attributeattribute correspondence $\left(a, b_{2}\right)$ form the same primitive correspondence $\left(\bar{a}, \overline{b_{2}}\right)$. These two types of correspondences usually have similar confidence. For example, an attributevalue correspondence may have high confidence 0.9 , but the corresponding attribute-attribute correspondence may have confidence 0.8 or even 0.95 . This tends to predict more correspondences, reducing precision. Relation-value correspondences have the same issue.

2. Duplicate methods cannot discover all correspondences with limited duplicate data. These methods determine correspondences from limited number of duplicate data ( 5 and 10 in our experiments). These limited number of duplicate data may not cover all cases of correspondences. In the Stock data set, the top duplicate data only cover company AAPL and GOOG, but no IBM. Thus, the correspondences involved IBM cannot be predicted, which reduces recall. One possible solution is to increase the number of duplicates. However, as the number of duplicates increases, there will be more errors in record linkage. A possible future work should select duplicate data by not only considering confidences, but also diversity in terms of generating correspondences.

3. The similarity measures in both duplicate and nonduplicate methods need improvement. The distinguishing capability of similarity measure is difficult to choose. For one extreme end, the similarity measure may only consider exactly matched data. For the other extreme end, the similarity measure may just consider the data types. Different distinguishing capability determines the balance between precision and recall. In our experiments, the implemented similarity measure performs well on the Stock and Game set, but poorly on the Ecommerce set for the duplicate methods and on the Enrollment set for the non-duplicate method. This indicates that different applications may need similarity measures with different capabilities.

\section{RELATED WORK}

\subsection{Schema Matching}

Schema matching is well studied [3, 13, 4]. Many existing systems exploit data instances to generate matchings, such as LSD [9], and GLUE [11]. Most instance-based schema matching systems extract the values of all tuples for each attribute as a set, and compute the confidence of a correspondence either by similarity measures or machine learning algorithms. The DUMAS system proposes another approach that first finds similar tuples, and determines schema matchings based on those tuples [5]. Although DUMAS has the limitation that the two databases need to share duplicate tuples, the experiments show that they only need a few duplicates (fewer than 10) to achieve reasonable accuracy. In schema matching literature, most systems define the matching task as finding correspondences between attributes. In this paper, we define three additional correspondence types as compound correspondences. Schema matchings may not be only determined by schemas, QODI uses both schema and user queries for matching [22].

The iMap system introduces a special attribute-value correspondence [8]. They focus on the case where target attributes have binary data values. We define the attributevalue correspondences for general cases. In addition, we also define relation-attribute and relation-value correspondences.

\subsection{Data Exchange}

Data exchange is the problem of transforming an instance of a source schema to an instance of the target schema, given the mappings between the two schemas [15, 1]. Clio is a state-of-the-art semi-automatic data exchange system [14]. Schema mappings in Clio are generated in two steps: finding initial matchings between attributes; and associating the matchings by logical inference through referential constraints. The matching association is done by a modification of the chase algorithm [20]. Using tgds as representation, our matchings can be used in Clio.

\subsection{Data Integration}

Data integration provides uniform access to a set of autonomous and heterogeneous structured data sources $[10$, $2]$. The schema mappings can be represented in three formalisms. Global-as-view (GAV) represents each relation in the target schema as a view of the source schemas. Local-asview (LAV) represents each relation in the source schemas as a view of the target schema [19]. The combination of GAV and LAV is Global-local-as-view (GLAV), which includes a mapping from a view of the target schema to a view of the source schemas [16]. Tgds are equivalent in expressiveness 
to GLAV formalism.

\subsection{Record Linkage}

Record linkage is the task of matching records that refer to the same entity across different data sources [6, 17]. It is also called duplicate detection if the task is within only one data source [12]. In the relational database context, the goal of record linkage is to find pairs or groups of tuples that refer to the same real-world identity.

Record linkage across two different schemas is more difficult than duplicate detection, because it is not clear which attribute value in one schema should be compared with which attribute value in the other. DUMAS considers a tuple as a set of strings, and scores a pair of tuples by a similarity measure of the two sets [5]. The similarity measure is the cosine similarity between two TFIDF feature vectors. Our proposed duplicate methods extend the record linkage algorithms in DUMAS to find duplicate schema tuples.

\section{CONCLUSIONS AND FUTURE WORK}

Schema matching is conventionally defined as finding correspondences between attributes. As shown in our real world data sets of four application domains, this conventional definition has limitations. We introduce three additional types of correspondences consisting relations, attributes, and data values. The two proposed algorithms can automatically generate these correspondences. The correspondences can be formulated to tgds, and used in data exchange and data integration applications.

Many potential research questions are emerged following this work. First, the proposed methods of generating attribute-value and relation-value correspondences are only concerned with matchings between schema and existing data. A future direction should predict correspondences containing unseen data. Second, a ranking algorithm of duplicate pairs considering both similarity and diversity is important to the duplicate methods. Third, the similarity measures should be improved, especially for non-string data types.

\section{REFERENCES}

[1] M. Arenas, P. Barcelo, L. Libkin, and F. Murlak. Relational and xml data exchange. Synthesis Lectures on Data Management, 2(1):1-112, 2010.

[2] F. Barbançon and D. Miranker. Sphinx: Schema integration by example. Journal of Intelligent Information Systems, 29(2):145-184, 2007.

[3] Z. Bellahsene, A. Bonifati, and E. Rahm, editors. Schema Matching and Mapping. Springer, 2011.

[4] P. Bernstein, J. Madhavan, and E. Rahm. Generic schema matching, ten years later. Proceedings of the VLDB Endowment, 4(11), 2011.

[5] A. Bilke and F. Naumann. Schema matching using duplicates. In Data Engineering, 2005. ICDE 2005. Proceedings. 21st International Conference on, pages 69-80. IEEE, 2005.

[6] P. Christen. A survey of indexing techniques for scalable record linkage and deduplication. Knowledge and Data Engineering, IEEE Transactions on, (99):1-1, 2011.
[7] W. Cohen, P. Ravikumar, and S. Fienberg. A comparison of string distance metrics for name-matching tasks. In Proceedings of the IJCAI2003 Workshop on Information Integration on the Web IIWeb03, pages 73-78, 2003.

[8] R. Dhamankar, Y. Lee, A. Doan, A. Halevy, and P. Domingos. imap: discovering complex semantic matches between database schemas. In SIGMOD 2004, pages 383-394. ACM, 2004.

[9] A. Doan, P. Domingos, and A. Halevy. Reconciling schemas of disparate data sources: A machine-learning approach. In SIGMOD 2001, pages 509-520. ACM, 2001.

[10] A. Doan, A. Halevy, and Z. Ives. Principles of Data Integration. Elsevier Science, 2012.

[11] A. Doan, J. Madhavan, R. Dhamankar, P. Domingos, and A. Halevy. Learning to match ontologies on the semantic web. The VLDB Journal, 12(4):303-319, 2003.

[12] A. Elmagarmid, P. Ipeirotis, and V. Verykios. Duplicate record detection: A survey. Knowledge and Data Engineering, IEEE Transactions on, 19(1):1-16, 2007.

[13] J. Euzenat and P. Shvaiko. Ontology matching. Springer-Verlag New York Inc, 2007.

[14] R. Fagin, L. Haas, M. Hernández, R. Miller, L. Popa, and Y. Velegrakis. Clio: Schema mapping creation and data exchange. Conceptual Modeling: Foundations and Applications, pages 198-236, 2009.

[15] R. Fagin, P. G. Kolaitis, R. J. Miller, and L. Popa. Data exchange: semantics and query answering. Theoretical Computer Science, 336(1):89-124, 2005.

[16] M. Friedman, A. Levy, and T. Millstein. Navigational plans for data integration. In Proceedings of the National Conference on Artificial Intelligence, pages 67-73, 1999.

[17] N. Koudas, S. Sarawagi, and D. Srivastava. Record linkage: similarity measures and algorithms. In Proceedings of the 2006 ACM SIGMOD international conference on Management of data, pages 802-803. ACM, 2006.

[18] R. Krishnamurthy, W. Litwin, and W. Kent. Language features for interoperability of databases with schematic discrepancies. SIGMOD 1991, 20(2):40-49, 1991.

[19] A. Y. Levy, A. Rajaraman, and J. J. Ordille. Querying heterogeneous information sources using source descriptions. In Proceedings of the 22th International Conference on Very Large Data Bases, pages 251-262. Morgan Kaufmann Publishers Inc., 1996.

[20] D. Maier, A. O. Mendelzon, and Y. Sagiv. Testing implications of data dependencies. ACM Trans. Database Systems (TODS), 4(4):455-469, 1979.

[21] R. Pottinger and A. Halevy. Minicon: A scalable algorithm for answering queries using views. The

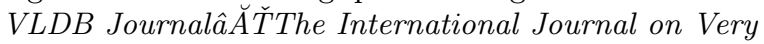
Large Data Bases, 10(2-3):182-198, 2001.

[22] A. Tian, J. Sequeda, and D. Miranker. Qodi: Query as context in automatic data integration. In 12th International Semantic Web Conference, pages 624-639. Springer Berlin Heidelberg, 2013. 